Review Article

\title{
Early Prognostic Utility of Gp210 Antibody-Positive Rate in Primary Biliary Cholangitis: A Meta-Analysis
}

\author{
Chunyang Huang $\mathbb{D}^{1,2}$ Weijia Han $\mathbb{D}^{1,3}$ Chuanmin Wang ${ }^{\mathbb{D}},{ }^{4}$ Yanmin Liu $\mathbb{D}^{1,2}$ Yue Chen ${ }^{1 D}{ }^{4}$ \\ and Zhongping Duan $\mathbb{1}^{1,3}$ \\ ${ }^{1}$ Difficult \& Complicated Liver Diseases and Artificial Liver Center, Beijing Youan Hospital, Capital Medical University, China \\ ${ }^{2}$ Department of Immunologic Liver Disease, Beijing Youan Hospital, Capital Medical University, Beijing, China \\ ${ }^{3}$ Beijing Municipal Key Laboratory of Liver Failure and Artificial Liver Treatment Research, Beijing, China \\ ${ }^{4}$ Department of Infectious Diseases, Taihe Hospital, Shiyan, Hubei, China
}

Correspondence should be addressed to Yue Chen; cyue63@126.com and Zhongping Duan; duan2517@163.com

Received 23 May 2019; Revised 15 July 2019; Accepted 12 August 2019; Published 13 October 2019

Academic Editor: Anja Hviid Simonsen

Copyright (C) 2019 Chunyang Huang et al. This is an open access article distributed under the Creative Commons Attribution License, which permits unrestricted use, distribution, and reproduction in any medium, provided the original work is properly cited.

\begin{abstract}
Background. The prevalence of primary biliary cholangitis (PBC), which is an autoimmune liver disease, has increased over time. PBC often leads to severe consequences, such as liver failure and death. Stratification tools using biochemical liver tests are needed to assess and predict the progression of this disease at the time of PBC diagnosis. Methods. We searched PubMed, Cochrane Library, Web of Science, and Embase for studies focused on the relationship between positive rates of Gp210 antibodies and poor prognosis of PBC. The primary end point was the number of PBC patients with poor outcome in the Gp210 antibody (+) and Gp210 antibody (-) groups. The secondary end point was the basic serum level of alanine aminotransferase (ALT), alkaline phosphatase (ALP), total bilirubin (TBIL), and IgM in the two groups. The age and number of female patients were also measured. Results. A total of 5 studies, comprising 737 patients, were included in this analysis. A positive rate of Gp210 antibodies was positively correlated with poor outcomes and with many types of progression in PBC, especially liver failure. Mortality was also higher in the Gp210 antibody (+) group. Furthermore, the serum levels of ALP and IgM were associated with the positive rate of Gp210 antibodies, while the serum levels of ALT and TBIL were not. The age and number of female patients were also not associated with the positive rate of Gp210 antibodies. Conclusion. PBC-specific Gp120 antibodies are optimal predictors of PBC prognosis at the time of diagnosis. Some other liver function indicators, such as ALP and IgM, can be used as predictors to complement Gp210 antibodies to establish a stratification tool to predict the prognosis of $\mathrm{PBC}$ at the time of diagnosis.
\end{abstract}

\section{Introduction}

Primary biliary cholangitis (PBC) is an autoimmune disease with an incidence of 0.9 to 5.8 per 100000 people worldwide. The prevalence of $\mathrm{PBC}$ has increased over time due to increased environmental triggers. PBC often leads to liver failure, cirrhosis, and even death. So, it is important to predict the progression of PBC. Although liver biopsy is the gold standard to assess the severity of $\mathrm{PBC}$, it is often limited by pain, invasiveness, interobserver disparity, and sampling error. Stratification tools, using biochemical liver tests applied after 1 year of ursodeoxycholic acid (UDCA) expo- sure, can readily identify patients with or without sufficient treatment response. For example, global score [1] and UK score [2] are useful for predicting PBC prognosis and the therapeutic effect of UDCA. However, these tools lack early predictive ability and cannot predict $\mathrm{PBC}$ prognosis at the time of diagnosis. So, a noninvasive, simple, and reliable method is needed to better assess and predict PBC progression at the time of diagnosis $[3,4]$.

Gp210 antibodies are highly specific for PBC. This type of antibody, with an integral glycoprotein of the nuclear pore complex, is typical of antinuclear antibodies. Some detection methods, such as a dual isotype ELISA, have been designed to 


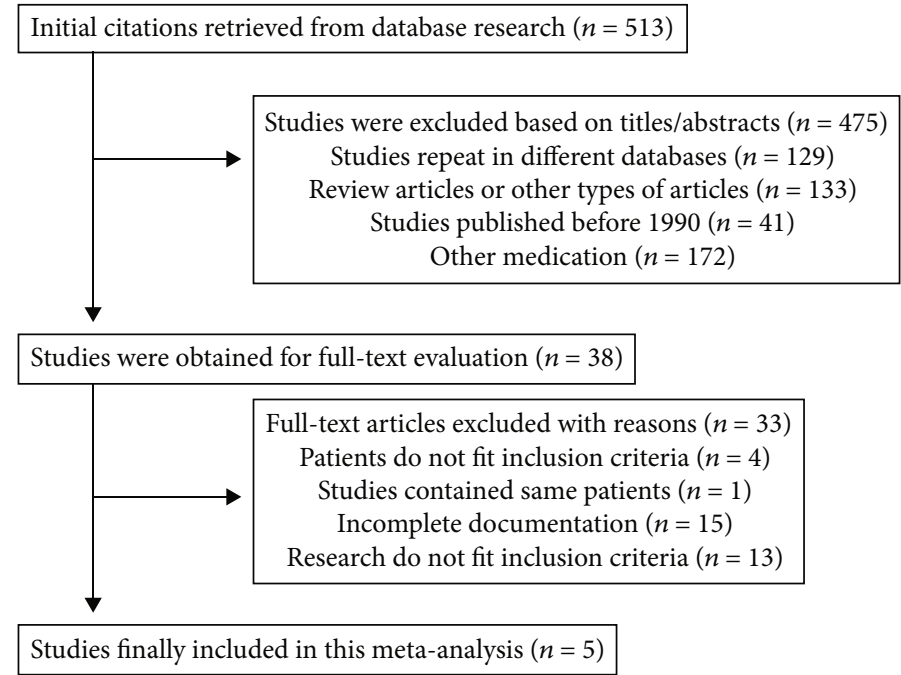

Figure 1: Flow diagram of study selection.

provide enhanced detection of Gp210 antibodies [5]. A metaanalysis has shown that Gp210 antibody positivity is an important diagnostic marker for PBC [6].

Some researchers have described the association between Gp210 antibodies and severe PBC prognosis. They have shown that Gp210 antibody (+) at the time of diagnosis is a strong risk factor for progression to end-stage hepatic failure and have described the clinical significance of Gp210 antibodies in monitoring PBC [7]. However, large samples and multicenter studies are needed to confirm the correlation between Gp210 antibody (+) rate and prognosis of PBC [8].

In this study, we summarize the currently published literature that has analyzed the relationship between Gp210 antibody (+) rate and prognosis of PBC. We aimed to evaluate the value of $\mathrm{Gp} 210$ antibodies in predicting poor prognosis of PBC at the time of PBC diagnosis. We also aimed to evaluate whether other liver function indicators at the time of $\mathrm{PBC}$ diagnosis can be used as predictors to complement Gp210 antibodies in predicting poor PBC prognosis. We hope to provide new ideas for further PBC management.

\section{Material and Methods}

We followed the methods of a published article by Yao et al. [9]. The processes of study retrieval and analysis were as follows.

2.1. Study Selection. This meta-analysis was conducted and reported according to the Preferred Reporting Items for Systematic Reviews and Meta-Analyses (PRISMA) [10]. We searched for articles from January 1990 to April 2019 using the databases of Cochrane Library, Web of Science, Embase, and PubMed. We selected all articles about prognosis of patients with PBC. The following search terms were used: (Primary biliary cirrhosis OR Liver Cirrhoses Biliary OR Biliary Cirrhosis OR Cirrhosis Biliary OR Secondary Biliary Cholangitis OR Biliary Cholangitis Secondary OR Cholangitis Secondary Biliary OR Secondary Biliary Cholangitides OR
Secondary Biliary Cirrhosis OR Cirrhosis, Secondary Biliary OR Biliary Cirrhosis Secondary OR Liver Cirrhosis, Obstructive OR Obstructive Liver Cirrhosis OR Primary Biliary Cholangitis OR Biliary Cholangitis Primary OR Cholangitis Primary Biliary OR Primary Biliary Cholangitides OR Biliary Cirrhosis Primary 1 OR Biliary Cirrhosis Primary OR Cholangitis Chronic Nonsuppurative Destructive OR Primary Biliary Cirrhosis) AND (prognosis OR Prognoses OR Prognostic Factors OR Factor Prognostic OR Factors Prognostic OR Prognostic Factor) AND (gp 210 OR gp210).

Two investigators (C.H. and W.H.) conducted a preliminary search separately, sifted through relevant headings and abstracts, deleted duplicate records, and identified relevant terms for further evaluation. References to retrieved articles were also reviewed to identify other eligible studies.

The Ethics Committee of Beijing Youan Hospital approved the study protocol.

2.2. Definition and Study End Points. PBC was diagnosed by increased antimitochondrial antibodies (AMAs) in a patient with increased alkaline phosphatase (ALP), assuming other intrahepatic and extrahepatic causes of cholestasis have been excluded [3]. This study contained two end points: (1) number of PBC patients with poor outcome in the Gp210 antibody (+) group and the Gp210 antibody (-) group. Adverse outcomes were defined as occurrence of PBC-related complications including ascites, variceal hemorrhage, hepatic encephalopathy, and high levels of total bilirubin (TBIL) [11], and (2) the serum levels of alanine aminotransferase (ALT), ALP, TBIL, and IgM in the Gp210 antibody (+) and Gp210 antibody (-) groups, and age and number of female patients in the two groups.

2.3. Data Extraction and Quality Assessment. Two investigators (C.H. and W.H.) extracted the following information independently from the selected studies: first author; year of publication; age and sex; number of patients enrolled in the Gp210 antibody (+) and Gp210 antibody (-) groups; number 


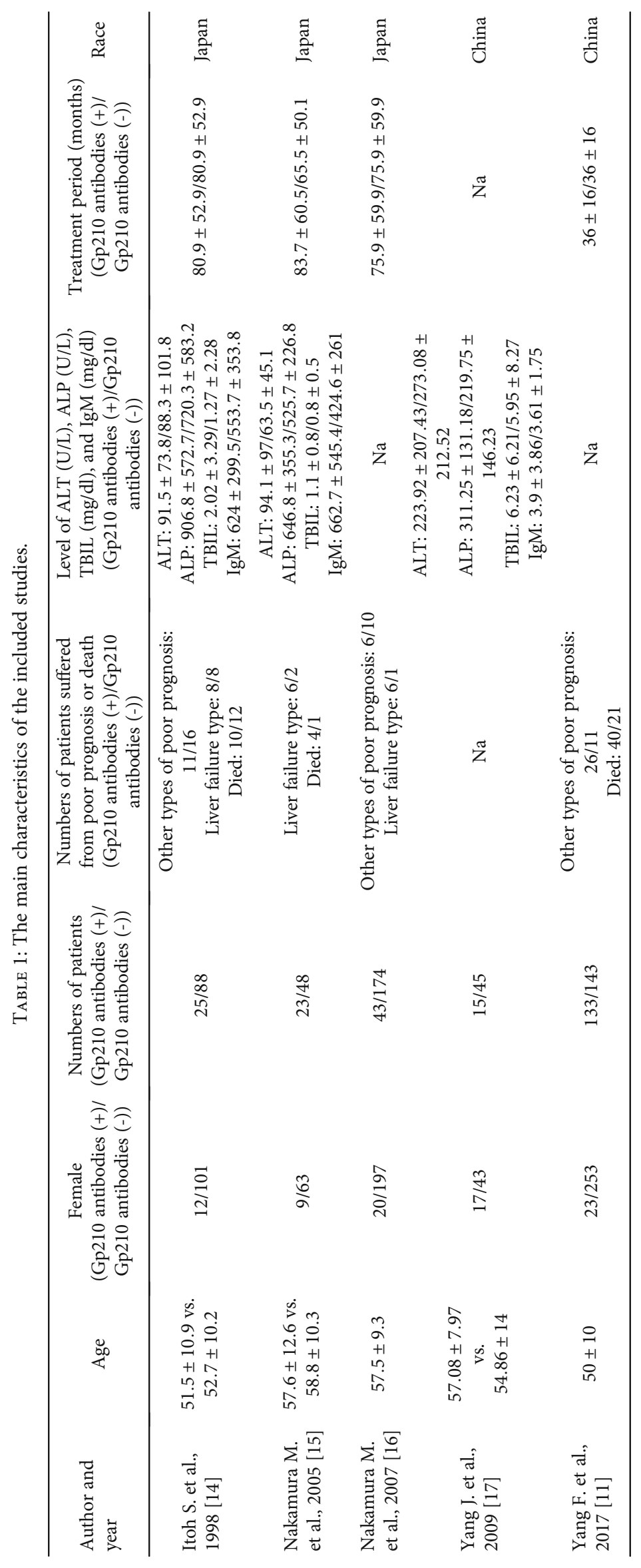




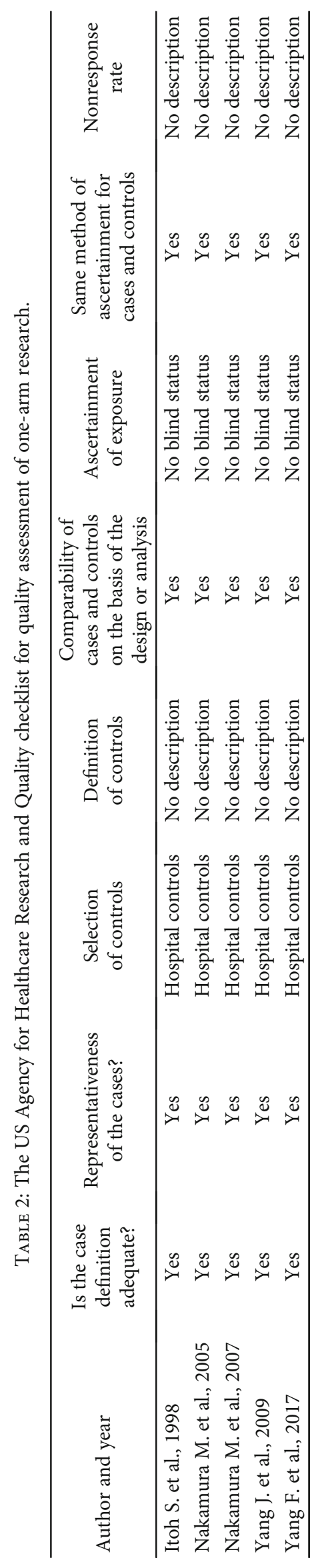




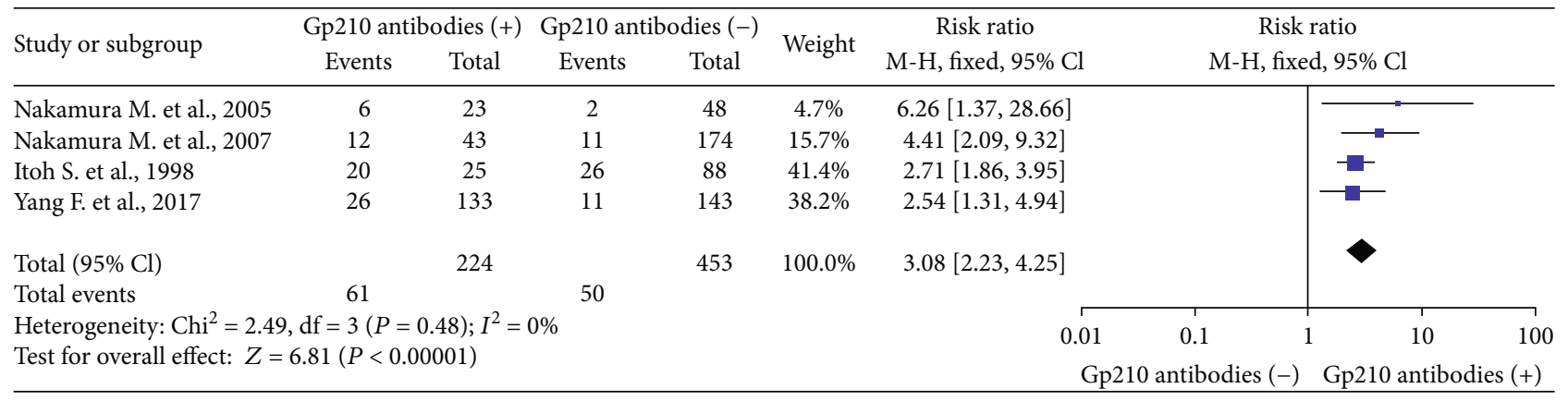

(a)

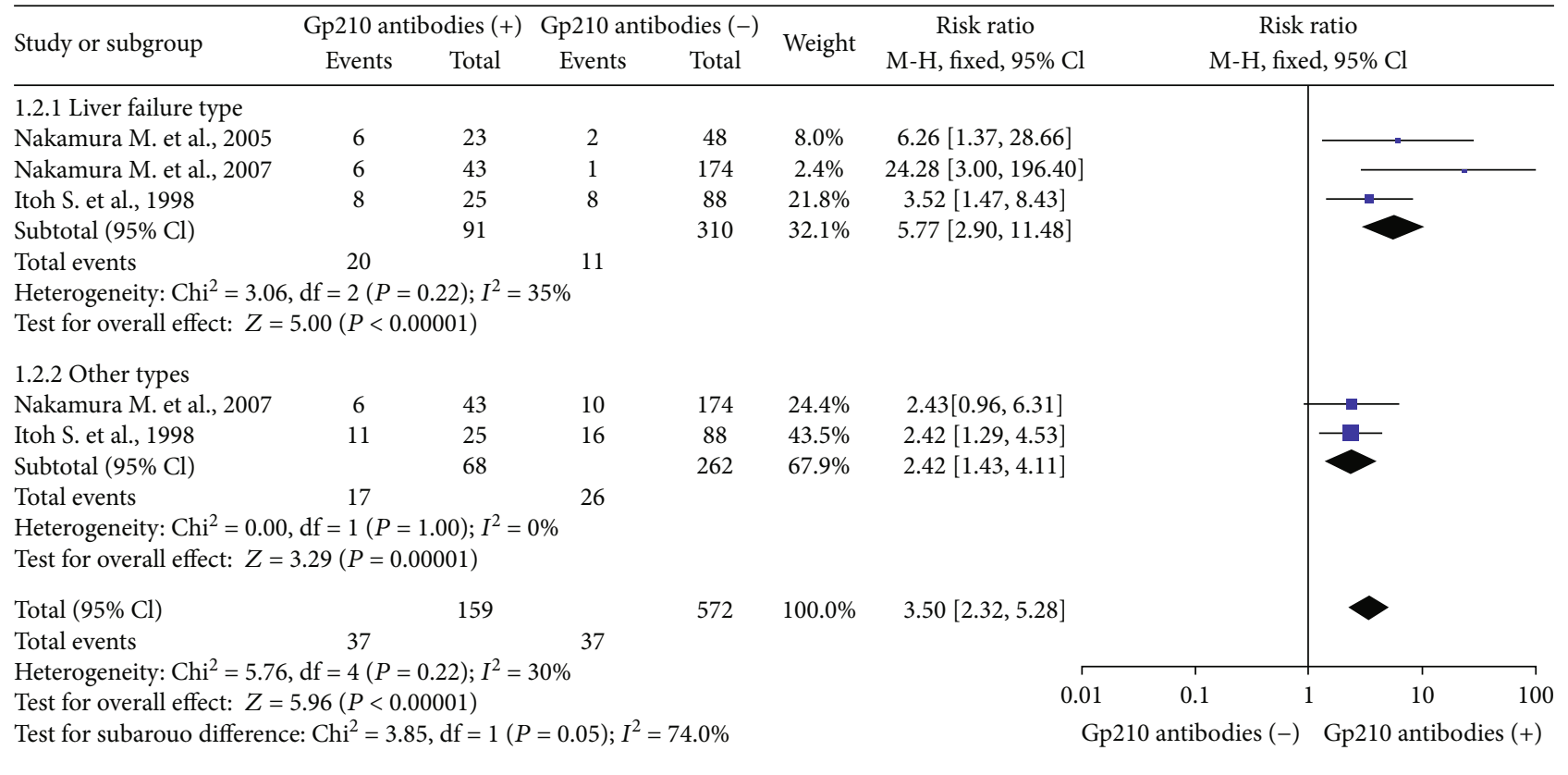

(b)

FIGURE 2: Meta-analysis of relationship between Gp210 antibody-positive rate and poor prognosis. (a) Comparisons of the incidence of poor prognosis between the Gp210 antibody (+) group and the Gp210 antibody (-) group. (b) Subgroup analysis of the incidence of different types of PBC progression.

of patients with poor prognosis, including adverse vital signs, liver failure, and death in the two groups; and the liver function indicators, including ALT, ALP, TBIL, and IgM in the two groups. When research on the same patients appeared in multiple articles, to avoid duplication of information, we selected the study with the largest sample.

The Newcastle-Ottawa scale (NOS) quality assessment was used to evaluate bias risks in each study.

2.4. Study Eligibility. Inclusion criteria were as follows [12, 13]: (1) chronic cholestasis after exclusion of other causes of liver disease, (2) unexplained elevation of serum ALP, (3) positivity of AMA, and (4) liver biopsy which was used to substantiate the diagnosis, but was rarely needed.

Exclusion criteria were as follows: positive serological test for hepatitis $\mathrm{B}$ or $\mathrm{C}$ virus and comorbidity of primary sclerosing cholangitis, alcoholic liver disease, hemochromatosis, Wilson's disease, a1-antitrypsin deficiency, and presence of complications of cirrhosis.
2.5. Statistical Analysis. We used Review Manager 5.2 and Stata 12.0 software for statistical analysis. Differences were expressed as relative risk (RR) with $95 \%$ confidence interval (CI) or standard mean difference (SMD) with 95\% CI. Heterogeneity was tested using the $I^{2}$ statistic. Heterogeneity was considered to be low in studies with $I^{2} 25-50 \%$, moderate in studies with $I^{2} 50-75 \%$, and high in studies with $I^{2}>75 \%$. $I^{2}>50 \%$ represented significant heterogeneity. A fixed-effects model was used when study heterogeneity was not significant, and a random-effects model when heterogeneity was significant. Begg's test was used to estimate publication bias, and sensitivity analysis to test stability.

\section{Results}

3.1. Study Selection and Characteristics. The selection process is illustrated in Figure 1. A total of 5 articles met the inclusion criteria [11, 14-17]. The main characteristics of the included studies are described in Table 1. The meta-analysis included 
Meta-analysis estimates, given named study is omitted

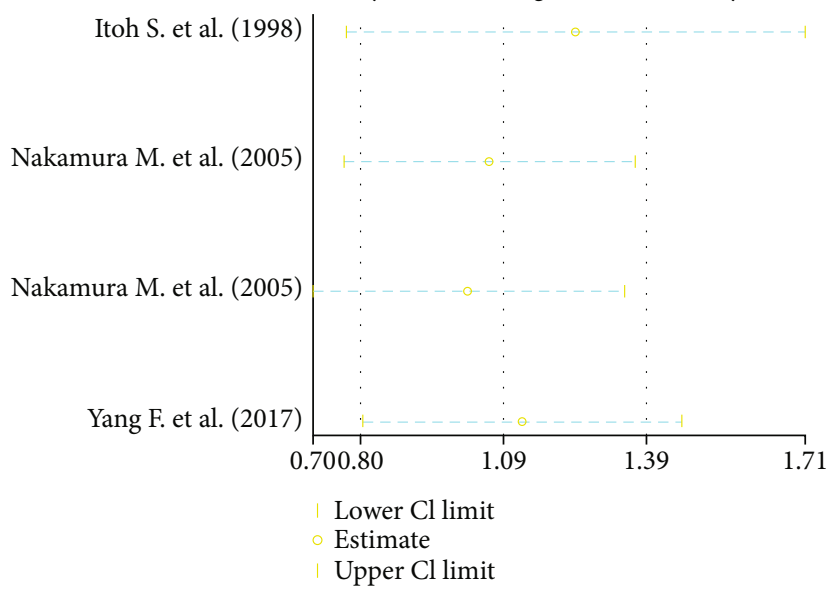

(a)

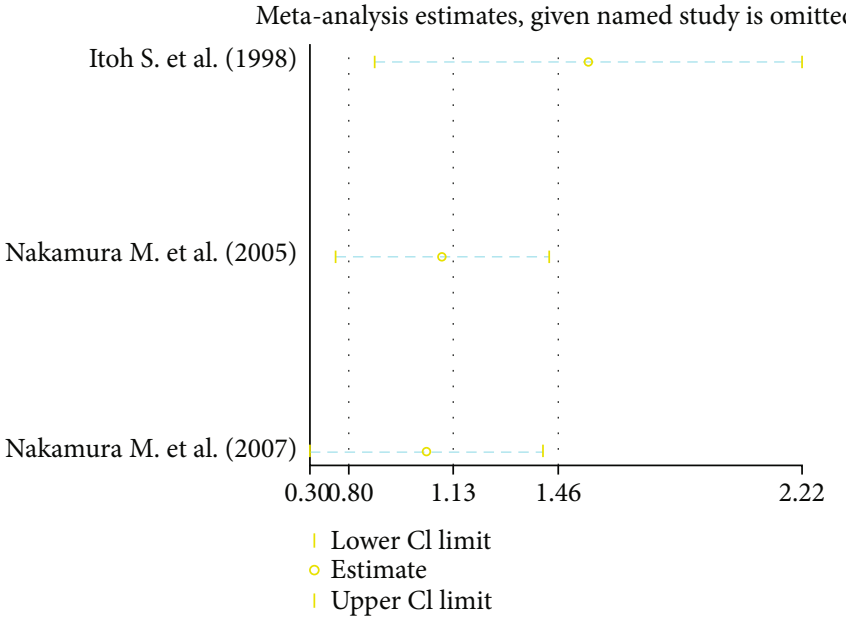

(b)

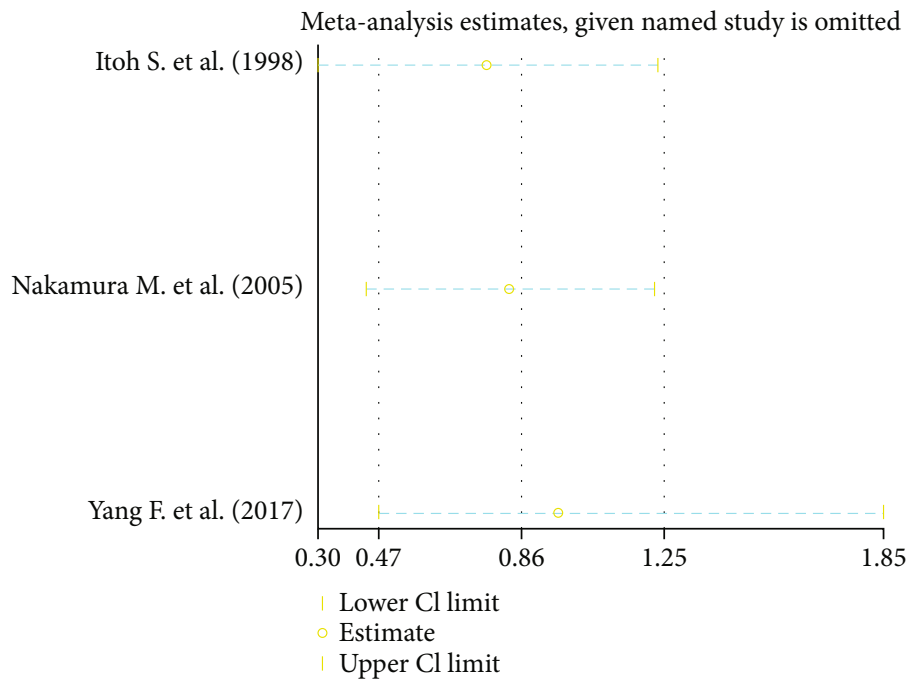

(c)

FIgURE 3: Sensitivity test for forest analysis. (a) Sensitivity analysis for Figure 2(a); (b) Sensitivity analysis for Figure 2(b); (c) Sensitivity analysis for Figure 5.

737 patients from East Asia, comprising 77 male and 660 female patients. There were 239 patients in the Gp210 antibody (+) group and 498 in the Gp210 antibody (-) group. Four of the 5 studies compared the incidence of poor prognosis between the two groups $[11,14-16]$. Three of the 5 studies compared death toll between the groups $[11,14,15]$. Three of the 5 studies compared the serum levels of ALT, ALP, TBIL, and IgM between the groups $[14,15,17]$.

3.2. Quality Assessment. All the studies were retrospective. The results of NOS quality assessment are shown in Table 2. The definition of case and control was all adequate, representative, and comparable. All the studies used the same method of exposure in the case and control groups. Ascertainment of exposure was conducted unblindedly.

3.3. Incidence of Poor Prognosis in the Gp210 Antibody (+) and Gp210 Antibody (-) Groups. We selected 4 studies that measured the incidence of poor prognosis in the
Gp210 antibody (+) and Gp210 antibody (-) groups [11, 14-16]. The incidence of poor prognosis was higher in the Gp210 antibody $(+)$ group $(\mathrm{RR}=3.08,95 \% \mathrm{CI}: 2.23-4.25)$. There was no heterogeneity $\left(I^{2}=6 \%\right)$ (Figure $\left.2(\mathrm{a})\right)$. Analysis of sensitivity confirmed the stability of this result $(1.09,95 \%$ CI: $0.8-1.39$ ), and the $95 \%$ CI for all articles was $0.7-1.71$ (Figure 3(a)). Begg's test showed publication bias in these 4 studies, although it was not significant $(\operatorname{Pr}>|z|=0.308$, continuity corrected) (Figure 4(a)).

Three of these 4 studies measured the incidence of different types of progression, such as liver failure, between the Gp210 antibody (+) and Gp210 antibody (-) groups $[11,14,16]$. We selected these 3 studies to make a subgroup analysis. The incidence of liver failure was higher in the Gp210 antibody $(+)$ group $(\mathrm{RR}=5.77,95 \% \mathrm{CI}: 2.9-11.48)$. The incidence of other types of progression was also higher in the Gp210 antibody $(+)$ group $(\mathrm{RR}=2.42,95 \% \mathrm{CI}$ : 1.43-4.11). The differences between the two groups were significant $(P=0.05)$ (Figure 2(b)). Analysis of sensitivity 


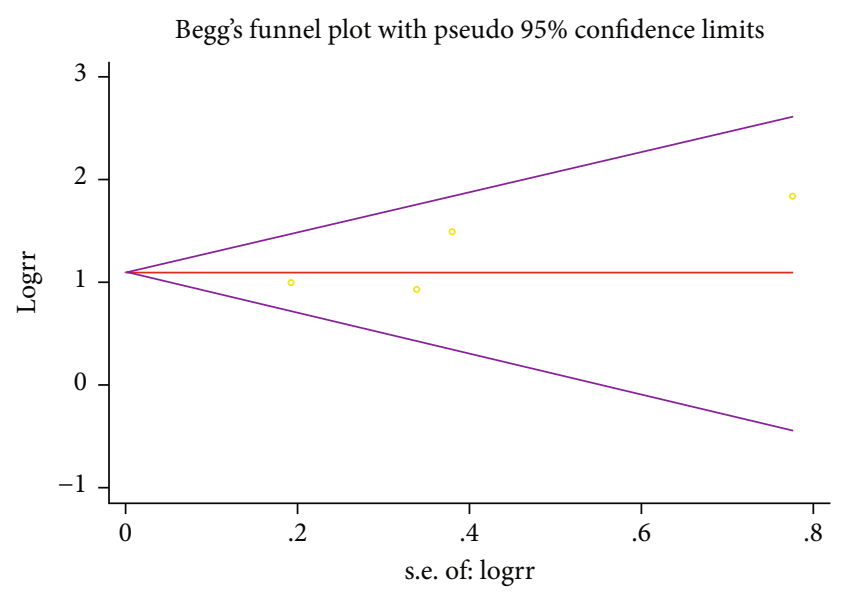

(a)

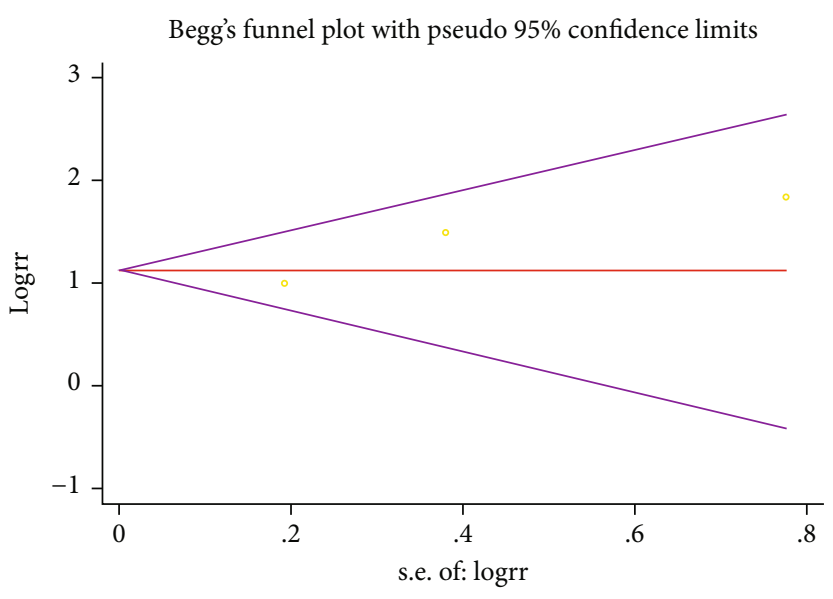

(b)

Begg's funnel plot with pseudo $95 \%$ confidence limits

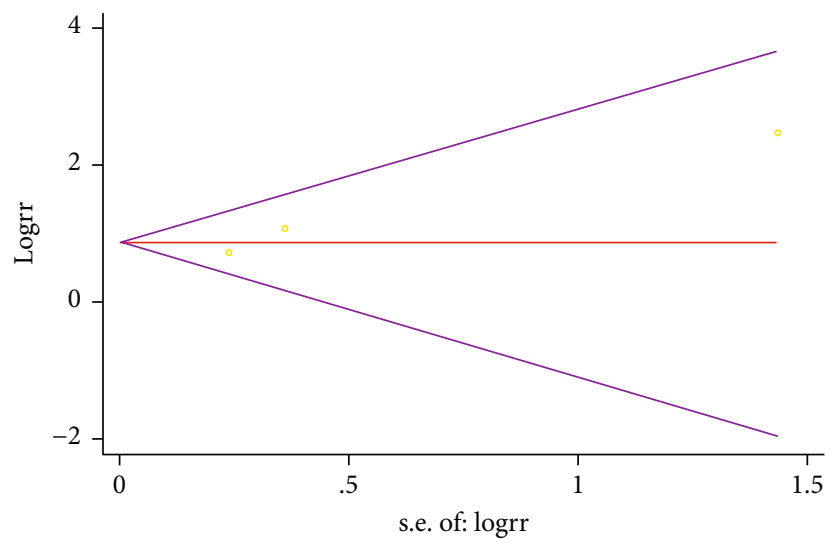

(c)

Figure 4: Begg's test for forest analysis. (a) Begg's test for Figure 2(a); (b) Begg's test for Figure 2(b); (c) Begg's test for Figure 5.

\begin{tabular}{|c|c|c|c|c|c|c|c|c|c|c|}
\hline \multirow{2}{*}{ Study or subgroup } & \multicolumn{2}{|c|}{ Gp210 antibodies $(+)$} & \multicolumn{2}{|c|}{ Gp210 antibodies (-) } & \multirow{2}{*}{ Weight } & \multirow{2}{*}{$\begin{array}{c}\text { Risk ratio } \\
\text { M-H, fixed, } 95 \% \mathrm{Cl}\end{array}$} & \multirow{2}{*}{\multicolumn{3}{|c|}{$\begin{array}{c}\text { Risk ratio } \\
\text { M-H, fixed, } 95 \% \mathrm{Cl}\end{array}$}} & \\
\hline & Events & Total & Events & Total & & & & & & \\
\hline Nakamura M. et al., 2005 & 4 & 23 & 1 & 48 & $2.5 \%$ & $8.35[0.99,70.54]$ & & & & \\
\hline Itoh S. et al., 1998 & 10 & 25 & 12 & 88 & $20.3 \%$ & $2.93[1.44,5.98]$ & & & & \\
\hline Yang F. et al., 2017 & 40 & 133 & 21 & 143 & $77.3 \%$ & $2.05[1.28,3.28]$ & & & & \\
\hline Total $(95 \% \mathrm{Cl})$ & & 181 & & 279 & $100.0 \%$ & $2.38[1.62,3.51]$ & & & & \\
\hline Total events & 54 & & 50 & & & & & & & \\
\hline \multirow{2}{*}{\multicolumn{6}{|c|}{$\begin{array}{l}\text { Heterogeneity: } \mathrm{Chi}^{2}=2.05, \mathrm{df}=3(P=0.36) ; I^{2}=2 \% \\
\text { Test for overall effect: } Z=4.40(P<0.00001)\end{array}$}} & 0.0 & 0.1 & 1 & 10 & 100 \\
\hline & & & & & & & ntibo & & 0 anti & es $(+)$ \\
\hline
\end{tabular}

FIGURE 5: Meta-analysis of relationship between Gp210 antibody-positive rate and incidence of mortality.

confirmed the stability of this result $(1.13,95 \%$ CI: $0.80-1.46)$, and the range for all articles was $0.68-2.22$ (Figure 3(b)). Begg's test showed publication bias in these 3 studies, although it was not significant $(\operatorname{Pr}>|z|=1$, continuity corrected) (Figure 4(b)).

3.4. Incidence of Mortality in the Gp210 Antibody (+) and Gp210 Antibody (-) Groups. We selected 3 studies that measured mortality in the Gp210 antibody (+) and Gp210 antibody (-) groups $[11,14,15]$. The mortality was higher in the Gp210 antibody $(+)$ group $(\mathrm{RR}=2.38$, 95\% CI: $1.62-$
3.51). There was no heterogeneity $\left(I^{2}=0 \%\right)$ (Figure 5$)$. Analysis of sensitivity evaluated the robustness of the effect $(0.86$, 95\% CI: 0.47-1.25), and the range for all articles was $0.3-1.85$ (Figure 3(c)). Begg's test showed publication bias in these 3 studies, although it was not significant $(\operatorname{Pr}>|z|=0.296$, continuity corrected) (Figure 4(c)).

3.5. Serum Levels of ALT, ALP, TBIL, and IgM in the Gp210 Antibody (+) and Gp210 Antibody (-) Groups. The serum levels of liver function and immune indicators, including TBIL, ALT, ALP, and IgM, were measured in the Gp210 


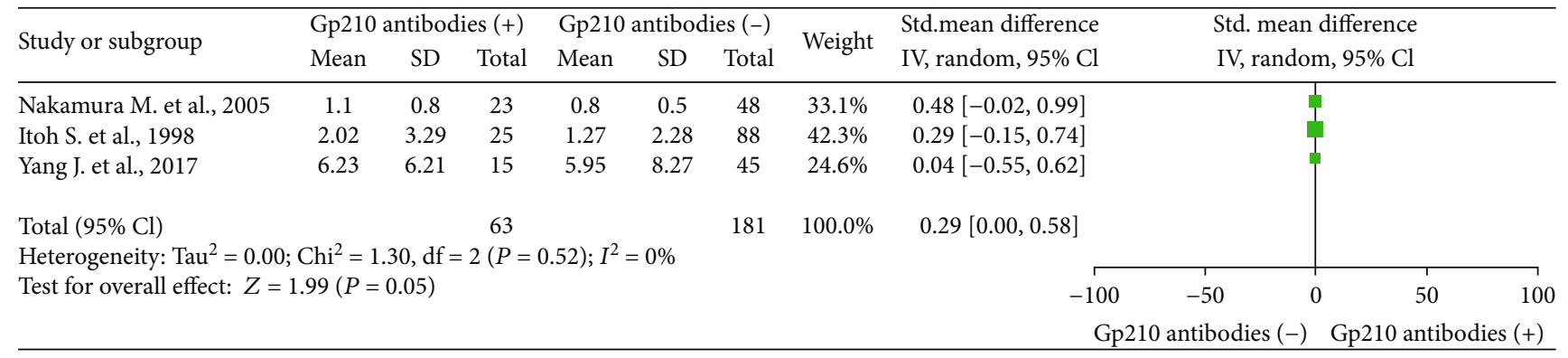

(a)

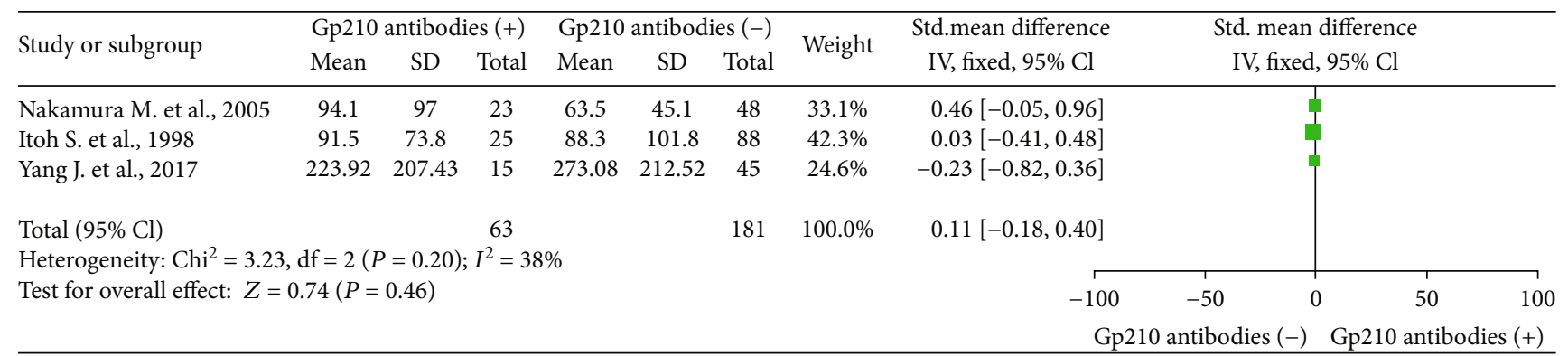

(b)

\begin{tabular}{|c|c|c|c|c|c|c|c|c|c|c|c|c|}
\hline \multirow{2}{*}{ Study or subgroup } & \multicolumn{3}{|c|}{ Gp210 antibodies (+) } & \multicolumn{3}{|c|}{ Gp210 antibodies $(-)$} & \multirow{2}{*}{ Weight } & \multirow{2}{*}{$\begin{array}{l}\text { Std.mean difference } \\
\text { IV, fixed, } 95 \% \mathrm{Cl}\end{array}$} & \multirow{2}{*}{\multicolumn{3}{|c|}{$\begin{array}{l}\text { Std. mean difference } \\
\text { IV, fixed, } 95 \% \mathrm{Cl}\end{array}$}} & \\
\hline & Mean & SD & Total & Mean & SD & Total & & & & & & \\
\hline Nakamura M. et al., 2005 & 646.8 & 355.3 & 23 & 525.7 & 226.8 & 48 & $33.1 \%$ & $0.44[-0.07,0.94]$ & & 1 & & \\
\hline Itoh S. et al., 1998 & 906.8 & 572.7 & 25 & 720.3 & 583.2 & 88 & $42.3 \%$ & $0.32[-0.13,0.77]$ & & & & \\
\hline Yang J. et al., 2017 & 311.25 & 131.18 & 15 & 219.75 & 146.23 & 45 & $24.6 \%$ & $-0.63[0.04,1.23]$ & & & & \\
\hline \multirow{3}{*}{\multicolumn{3}{|c|}{$\begin{array}{l}\text { Total }(95 \% \mathrm{Cl}) \\
\text { Heterogeneity: } \mathrm{Chi}^{2}=0.68, \mathrm{df}=2(P=0.71) \text {; } \\
\text { Test for overall effect: } Z=2.92(P=0.004)\end{array}$}} & $\begin{array}{c}63 \\
I^{2}=0\end{array}$ & & & 181 & $100.0 \%$ & $0.11[-0.18,0.40]$ & & & & \\
\hline & & & & & & & & -100 & -50 & 0 & 50 & 100 \\
\hline & & & & & & & & Gp & intibo & & 0 antib & $s(+)$ \\
\hline
\end{tabular}

(c)

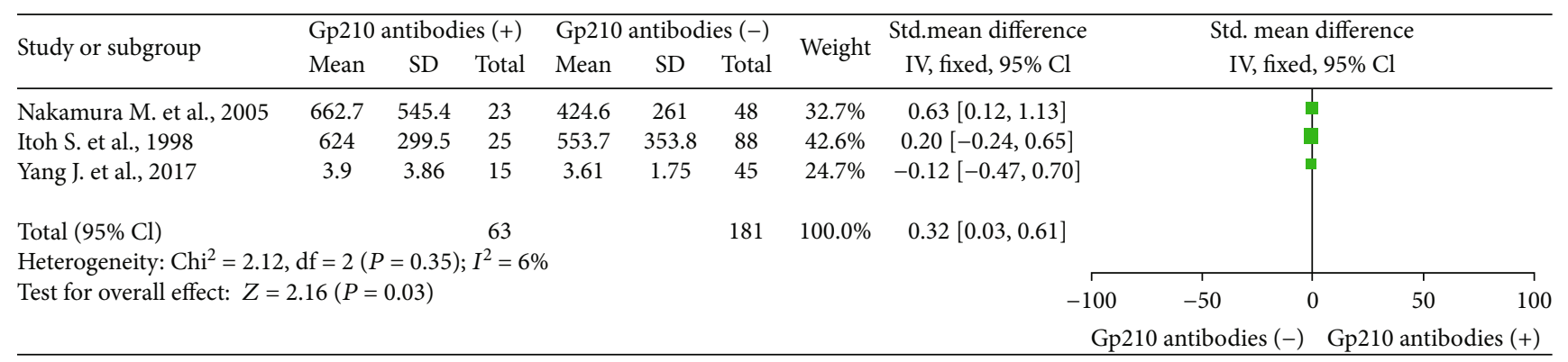

(d)

Figure 6: Meta-analysis of relationship between Gp210 antibody-positive rate and liver function indicators. (a) TBIL; (b) ALT; (c) ALP; (d) IgM.

antibody (+) and Gp210 antibody (-) groups [14, 16, 17]. For liver function indicators, there was no significant difference in the serum level of TBIL between the groups $(\mathrm{SMD}=0.29$, $95 \%$ CI $0-0.58)$, and there was no heterogeneity $\left(I^{2}=0 \%\right)$ (Figure 6(a)). Similarly, there was no significant difference in the serum level of ALT between the groups $(\mathrm{SMD}=0.11$, 95\% CI: -0.18 to 0.40$)$. There was moderate heterogeneity $\left(I^{2}=38 \%\right)$ (Figure 6(b)). The serum level of ALP was higher in the Gp210 antibody $(+)$ group $(\mathrm{SMD}=0.43,95 \% \mathrm{CI}$ : $0.14-0.72)$. There was no significant heterogeneity $\left(I^{2}=0 \%\right)$
(Figure 6(c)). For immune indicators, there was a significant difference in the serum level of IgM $(\mathrm{SMD}=0.32,95 \% \mathrm{CI}$ : 0.03-0.61) (Figure 6(d)) between the two groups.

3.6. Age and Number of Female Patients in the Gp210 Antibody (+) and Gp210 Antibody (-) Groups. The age and number of female patients were measured in the Gp210 antibody (+) and Gp210 antibody (-) groups [14, 16, 17]. There was no significant difference in age between the groups $(\mathrm{SMD}=-0.04,95 \% \mathrm{CI}:-0.33$ to 0.25$)$, and there was no 


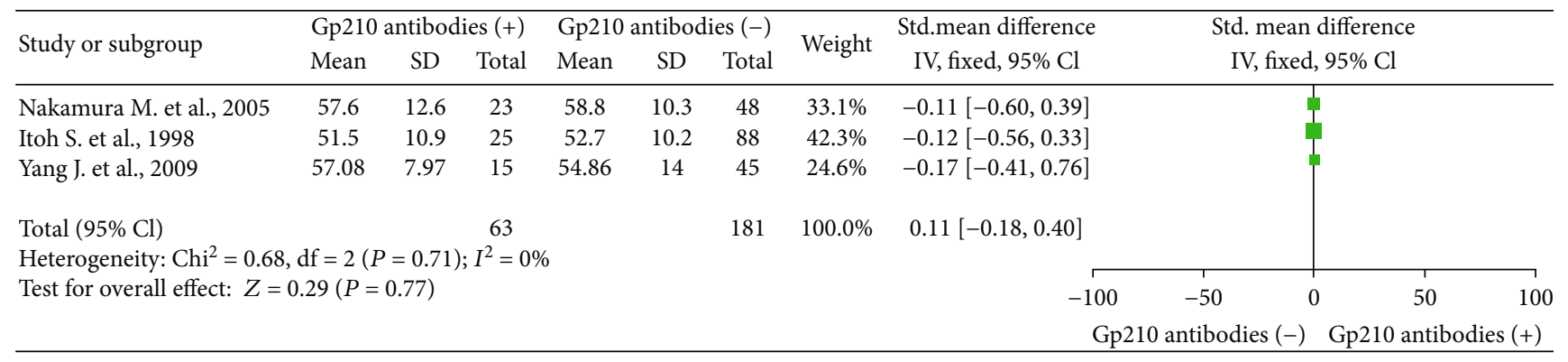

(a)

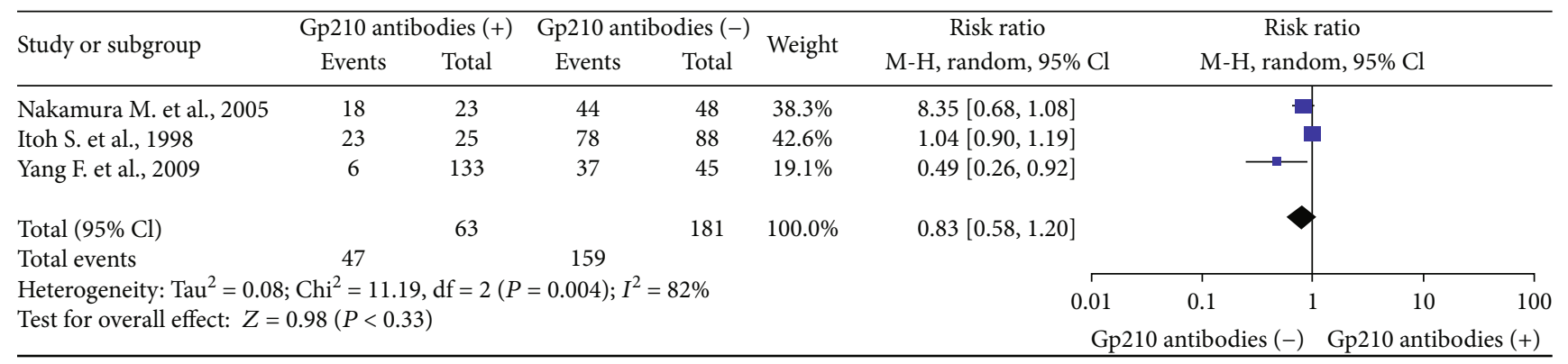

(b)

FIGURE 7: Meta-analysis of relationship between Gp210 antibody-positive rate and age or sex. (a) age; (b) sex.

heterogeneity $\left(I^{2}=0 \%\right.$ ) (Figure $7(\mathrm{a})$ ). Similarly, there was no significant difference in sex between the groups $(\mathrm{RR}=$ 0.83, 95\% CI: $0.58-1.20)$ with high heterogeneity $\left(I^{2}=82 \%\right)$ (Figure 7(b)).

\section{Discussion}

This study evaluated the published literature on the positive rate of Gp210 antibodies in anticipating the poor prognosis of $\mathrm{PBC}$ at the time of diagnosis. The results support that the positive rate of Gp210 antibodies is positively correlated with poor prognosis and even positively correlated with the mortality rate. Furthermore, we found that the basal level of some indicators, including ALP and IgM, are higher in the Gp210 antibody (+) group. Our results provide evidence for Gp210 antibodies as an early prognostic indicator of PBC. Combination of Gp210 antibodies, ALP and IgM may be a good prognostic tool for $\mathrm{PBC}$ at the time of diagnosis in the future.

Gp210 antibodies have been reported as highly specific for PBC. The roles of Gp210 antibodies in PBC are as follows. Bacterial components and other environmental triggers may be involved in the pathogenesis of PBC. These triggers, for example, bacterial lipoteichoic acid and histonelike DNA-binding protein, are detectable by synthetic Gp210 antibodies. These Gp210 antibodies, whose target antigen is a $210 \mathrm{kDa}$ transmembrane glycoprotein located on the nuclear pore complex, act against an approximately $210 \mathrm{kDa}$ polypeptide of the nuclear envelope. Gp210 antibodies promote apoptosis and autoantigen diffusion, break down immunological tolerance, and trigger PBC-like cholangitis, multifocal epithelial inflammation, and autoantibody production. Gp120 antibodies also sequentially upregulate innate and acquired immune responses, accompanied by autophagy and trigger nonsuppurative destructive cholangitis (Figure 8). It is widely known that the expression of Gp210 antibodies is increased on the nuclear envelope of biliary epithelial cells in small bile ducts in almost all specimens from PBC but is weak in autoimmune hepatitis and other autoimmune diseases. The level of Gp210 antibodies is positively correlated with portal inflammation, interface hepatitis, and lobular inflammation in PBC [16, 18-23].

In addition to widespread acknowledgement of their role in PBC diagnosis [24], many researchers have explored the important role of Gp210 antibodies in PBC prognosis in recent years. Nakamura et al. indicated that the increased expression of gp210 in small bile ducts, which is probably associated with inflammatory damage, is possibly involved in autoimmune response to gp210, leading to progression to end-stage hepatic failure in PBC [21]. At present, however, a large multicenter study is needed to confirm the prognostic utility of Gp210 antibodies. Our meta-analysis supported the idea that Gp210 antibodies at diagnosis are closely related to poor prognosis of PBC. Besides poor outcome, PBC has many types of progression, such as portal hypertension, liver failure, and jaundice. There are reports that liver failure in PBC is characterized by the presence of Gp210 antibodies, but other types of progression may not be so [25]. All types of PBC progression had a higher incidence in the Gp210 antibody $(+)$ group. Compared with other types of progression, the incidence of liver failure was significantly correlated with Gp210 antibodies. The mortality in the Gp210 antibody (+) group was also significantly higher than that in the Gp210 antibody (-) group.

The serum levels of liver function indicators (such as aminotransferase, albumin, and TBIL), age, and sex are recognized 


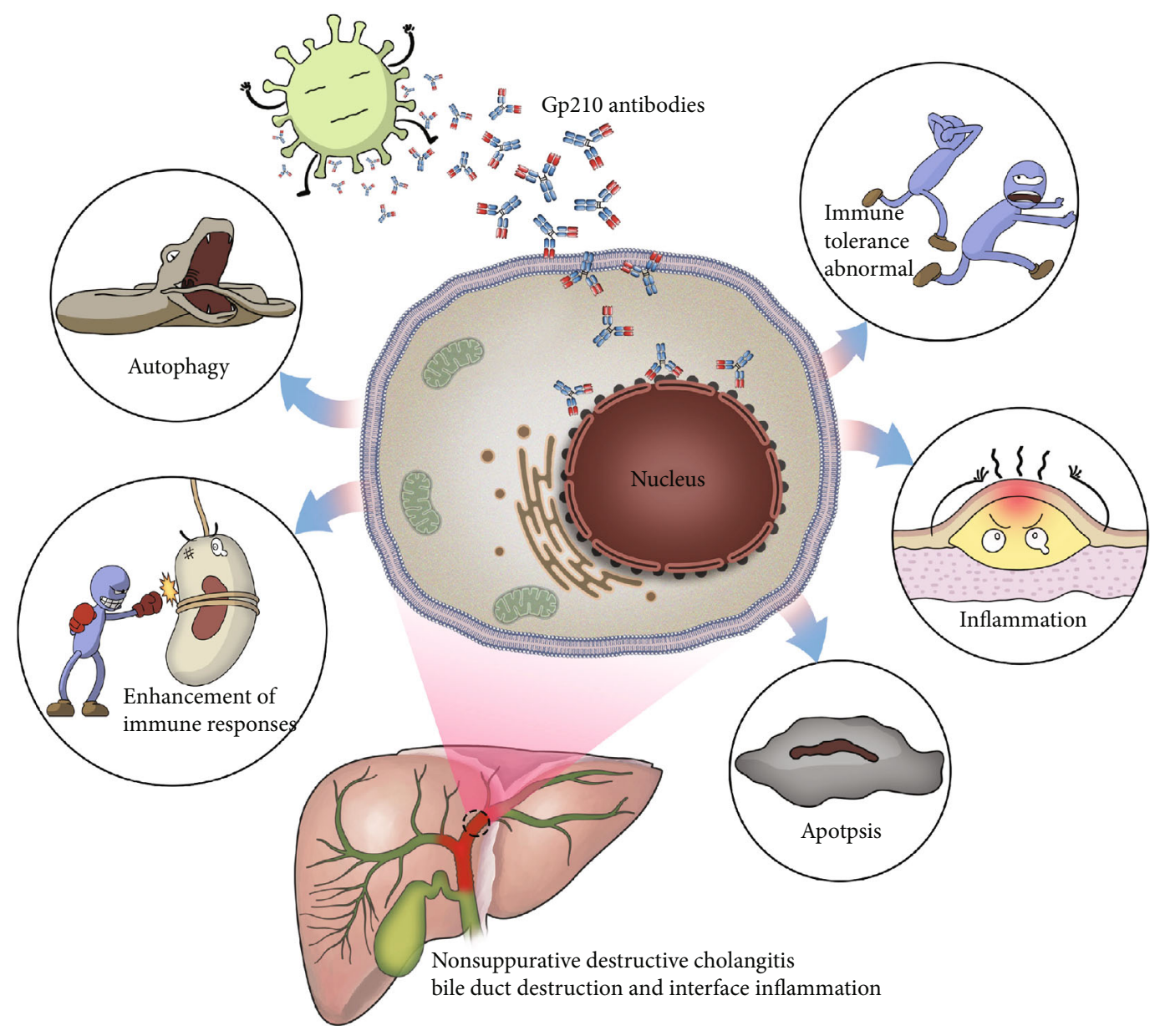

Figure 8: Roles of Gp210 antibodies in PBC.

as important predictors of survival in PBC after UDCA treatment $[26,27]$. One study has demonstrated that the serum level of immunoglobulin can estimate a more precise probability of survival for any given patient at any time during the course of the disease [28]. Whether these indicators can predict the prognosis of PBC with Gp210 antibodies at the time of $\mathrm{PBC}$ diagnosis has not yet been studied. In the present study, we found that serum levels of ALP and IgM were associated with Gp210 antibodies, while serum levels of ALT and TBIL were not. Sex and age were also not associated with Gp210 antibodies. Therefore, higher levels of ALP and IgM at diagnosis are two other predictors for poor prognosis of PBC. These indicators and Gp210 antibodies can be used in predicting the prognosis of $\mathrm{PBC}$ at the time of diagnosis. This provides a good basis for further PBC management.

There were some limitations to our study. First, there were only 5 studies that mentioned the relationship between Gp210 antibodies and PBC prognosis, and most of them had small samples. Second, some liver function indicators were not detected in these studies, such as $\gamma$-glutamyl transpeptadase. Third, the patients included in this study were all Asians. However, with the development of technology, new assay methods can enhance the detection of Gp210 antibodies. More high-quality studies are required to further analyze the effects of Gp210 antibodies in the prognosis of PBC.

\section{Conclusion}

PBC-specific Gp120 antibodies are optimal predictors of PBC prognosis at the time of diagnosis. Some other liver function indicators, such as ALP and IgM, can be used as predictors to complement Gp210 antibodies to establish a stratification tool to predict the prognosis of $\mathrm{PBC}$ at the time of diagnosis.

\section{Conflicts of Interest}

The authors declare no competing interests.

\section{Authors' Contributions}

Chunyang Huang and Weijia Han conduct preliminary search, extract information, calculate information, and write the paper; Chuanmin Wang revises the paper. Yanmin Liu designs the experiment. Zhongping Duan and Yue Chen approve the final version. Chunyang Huang and Weijia Han contributed equally to this work.

\section{Acknowledgments}

This article was supported by (1) National Key R\&D Program of China (No. 2017YFA0103000), (2) Beijing 
Municipal Administration of Hospitals Clinical Medicine Development of Special Funding Support (No. ZYLX201806), (3) "Beijing Municipal Administration of Hospitals" Ascent Plan (No. DFL20151601), (4) National Science and Technology Key Project on "Major Infectious Diseases such as HIV/AIDS, Viral Hepatitis Prevention and Treatment" (Nos. 2012ZX10002004-006, 2017ZX10203201005, 2017ZX10201201-001-001, 2017ZX10201201-002-002, 2017ZX10202203-006-001, and 2017ZX10302201-004-002), (5) project supported Beijing Municipal Administration of Hospitals Incubating Program (PX2019062), and (6) Key Medical Professional Development Plan of Beijing Municipal Administration of Hospitals (ZYLX201711).

\section{References}

[1] W. J. Lammers, G. M. Hirschfield, C. Corpechot et al., "Development and validation of a scoring system to predict outcomes of patients with primary biliary cirrhosis receiving ursodeoxycholic acid therapy," Gastroenterology, vol. 149, no. 7, pp. 1804-1812.e4, 2015.

[2] M. Carbone, S. J. Sharp, S. Flack et al., "The UK-PBC risk scores: derivation and validation of a scoring system for long-term prediction of end-stage liver disease in primary biliary cholangitis," Hepatology, vol. 63, no. 3, pp. 930-950, 2016.

[3] E. J. Carey, A. H. Ali, and K. D. Lindor, "Primary biliary cirrhosis," The Lancet, vol. 386, no. 10003, pp. 1565-1575, 2015.

[4] T. Umemura, S. Joshita, T. Sekiguchi et al., "Serum Wisteria floribunda agglutinin-positive Mac-2-binding protein level predicts liver fibrosis and prognosis in primary biliary cirrhosis," The American Journal of Gastroenterology, vol. 110, no. 6, pp. 857-864, 2015.

[5] H. Liu, G. L. Norman, Z. Shums et al., "PBC screen: an IgG/IgA dual isotype ELISA detecting multiple mitochondrial and nuclear autoantibodies specific for primary biliary cirrhosis," Journal of Autoimmunity, vol. 35, no. 4, pp. 436-442, 2010.

[6] S. L. Hu, F. R. Zhao, Q. Hu, and W. X. Chen, "Meta-analysis assessment of GP210 and SP100 for the diagnosis of primary biliary cirrhosis," PLoS One, vol. 9, no. 7, article e101916, 2014.

[7] M. Nakamura, "Clinical significance of autoantibodies in primary biliary cirrhosis," Seminars in Liver Disease, vol. 34, no. 3, pp. 334-340, 2014.

[8] European Association for the Study of the Liver, "EASL Clinical Practice Guidelines: the diagnosis and management of patients with primary biliary cholangitis," Journal of Hepatology, vol. 67, no. 1, pp. 145-172, 2017.

[9] J. Yao, W. Han, X. Ren, L. Yuan, J. Xu, and Z. Duan, "Improvement of energy substrate metabolism by late evening snack supplementation in patients with liver cirrhosis: a meta-analysis," Therapeutics and Clinical Risk Management, vol. 15, pp. 659-668, 2019.

[10] A. Pares, L. Caballeria, and J. Rodes, "Excellent long-term survival in patients with primary biliary cirrhosis and biochemical response to ursodeoxycholic acid," Gastroenterology, vol. 130, no. 3, pp. 715-720, 2006.

[11] F. Yang, Y. Yang, Q. Wang et al., "The risk predictive values of UK-PBC and GLOBE scoring system in Chinese patients with primary biliary cholangitis: the additional effect of anti-gp210," Alimentary Pharmacology \& Therapeutics, vol. 45, no. 5, pp. 733-743, 2017.
[12] K. D. Lindor, C. L. Bowlus, J. Boyer, C. Levy, and M. Mayo, "Primary biliary cholangitis: 2018 practice guidance from the American Association for the Study of Liver Diseases," Hepatology, vol. 69, no. 1, pp. 394-419, 2019.

[13] E. J. Heathcote, "Management of primary biliary cirrhosis," Hepatology, vol. 31, no. 4, pp. 1005-1013, 2000.

[14] S. Itoh, T. Ichida, T. Yoshida et al., "Autoantibodies against a $210 \mathrm{kDa}$ glycoprotein of the nuclear pore complex as a prognostic marker in patients with primary biliary cirrhosis," Journal of Gastroenterology and Hepatology, vol. 13, no. 3, pp. 257-265, 1998.

[15] M. Nakamura, Y. Shimizu-Yoshida, Y. Takii et al., “Antibody titer to gp210-C terminal peptide as a clinical parameter for monitoring primary biliary cirrhosis," Journal of Hepatology, vol. 42, no. 3, pp. 386-392, 2005.

[16] M. Nakamura, H. Kondo, T. Mori et al., “Anti-gp210 and anticentromere antibodies are different risk factors for the progression of primary biliary cirrhosis," Hepatology, vol. 45, no. 1, pp. 118-127, 2007.

[17] J. Yang, J. H. Yang, L. Y. You, Z. Y. Xu, and L. H. Yang, "Investigating the relationship between anti-gp210 antibody and clinical basic profile of primary billiary cirrhosis," Zhonghua Gan Zang Bing Za Zhi= Zhonghua Ganzangbing Zazhi= Chinese Journal of Hepatology, vol. 17, no. 6, pp. 468-469, 2009.

[18] I. Haruta, K. Kikuchi, M. Nakamura et al., "Involvement of commensal bacteria may lead to dysregulated inflammatory and autoimmune responses in a mouse model for chronic nonsuppurative destructive cholangitis," Journal of Clinical Immunology, vol. 32, no. 5, pp. 1026-1037, 2012.

[19] I. Haruta, K. Kikuchi, E. Hashimoto et al., "Long-term bacterial exposure can trigger nonsuppurative destructive cholangitis associated with multifocal epithelial inflammation," Laboratory Investigation, vol. 90, no. 4, pp. 577-588, 2010.

[20] I. Haruta, K. Kikuchi, E. Hashimoto et al., "A possible role of histone-like DNA-binding protein of Streptococcus intermedius in the pathogenesis of bile duct damage in primary biliary cirrhosis," Clinical Immunology, vol. 127, no. 2, pp. 245-251, 2008.

[21] M. Nakamura, Y. Takii, M. Ito et al., "Increased expression of nuclear envelope gp210 antigen in small bile ducts in primary biliary cirrhosis," Journal of Autoimmunity, vol. 26, no. 2, pp. 138-145, 2006.

[22] T. A. Ferguson, P. M. Stuart, J. M. Herndon, and T. S. Griffith, "Apoptosis, tolerance, and regulatory T cells - old wine, new wineskins," Immunological Reviews, vol. 193, no. 1, pp. 111123, 2003.

[23] S. Shimoda, M. Nakamura, H. Ishibashi et al., "Molecular mimicry of mitochondrial and nuclear autoantigens in primary biliary cirrhosis," Gastroenterology, vol. 124, no. 7, pp. 1915-1925, 2003.

[24] V. I. Reshetnyak, "Primary biliary cirrhosis: clinical and laboratory criteria for its diagnosis," World Journal of Gastroenterology, vol. 21, no. 25, pp. 7683-7708, 2015.

[25] Y. Takeshita, T. Takamura, O. Inoue et al., "Slowly progressive insulin-dependent diabetes in a patient with primary biliary cirrhosis with portal hypertension-type progression," Internal Medicine, vol. 51, no. 1, pp. 79-82, 2012.

[26] P. C. J. ter Borg, S. W. Schalm, B. E. Hansen, and H. R. van Buuren, "Prognosis of ursodeoxycholic acid-treated patients with primary biliary cirrhosis. Results of a 10-yr cohort study 
involving 297 patients," The American Journal of Gastroenterology, vol. 101, no. 9, pp. 2044-2050, 2006.

[27] E. R. Dickson, P. M. Grambsch, T. R. Fleming, L. D. Fisher, and A. Langworthy, "Prognosis in primary biliary cirrhosis: model for decision making," Hepatology, vol. 10, no. 1, pp. 1-7, 1989.

[28] E. Christensen, D. G. Altman, J. Neuberger et al., "Updating prognosis in primary biliary cirrhosis using a time-dependent Cox regression model," Gastroenterology, vol. 105, no. 6, pp. 1865-1876, 1993. 


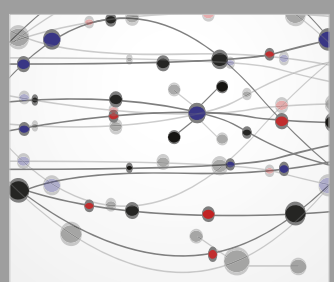

The Scientific World Journal
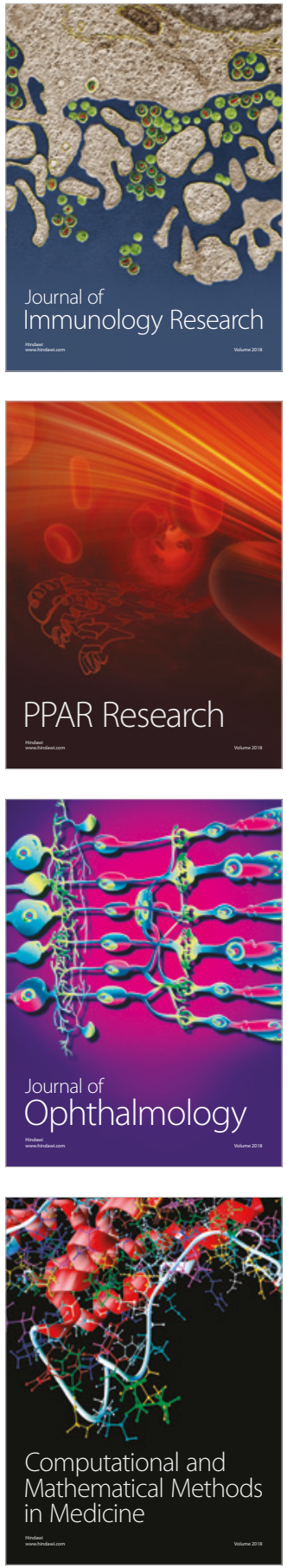

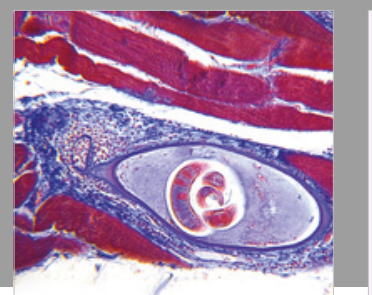

Gastroenterology Research and Practice

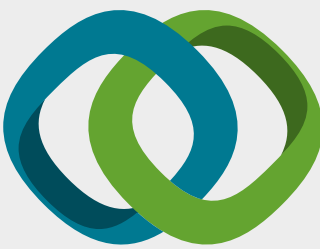

\section{Hindawi}

Submit your manuscripts at

www.hindawi.com
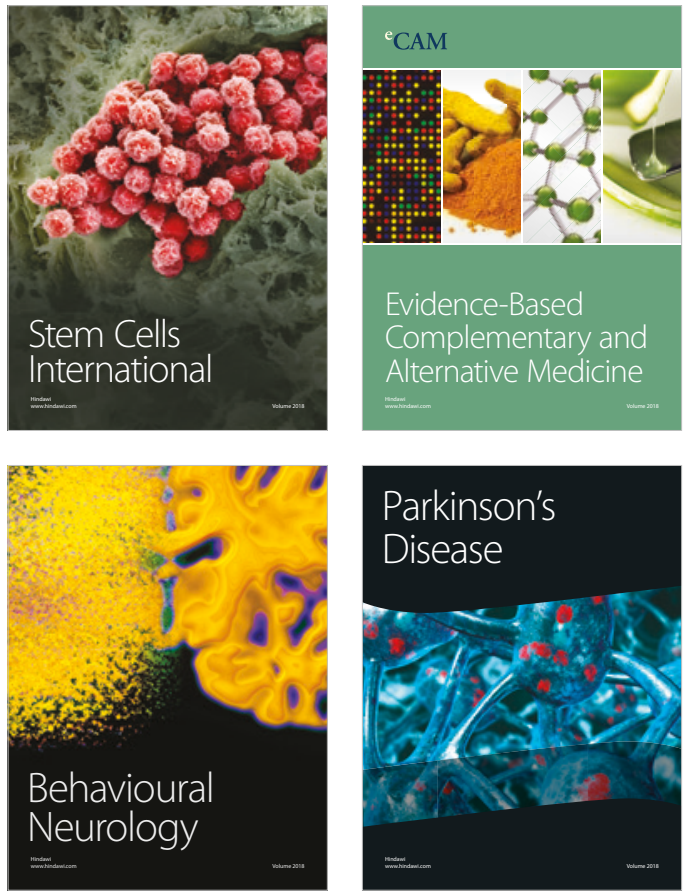

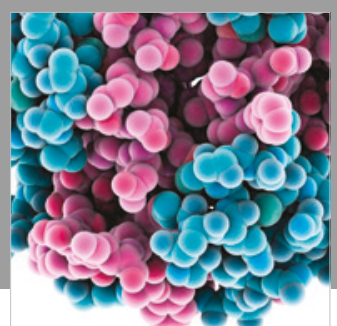

ournal of

Diabetes Research

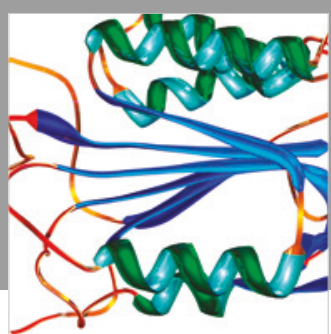

Disease Markers
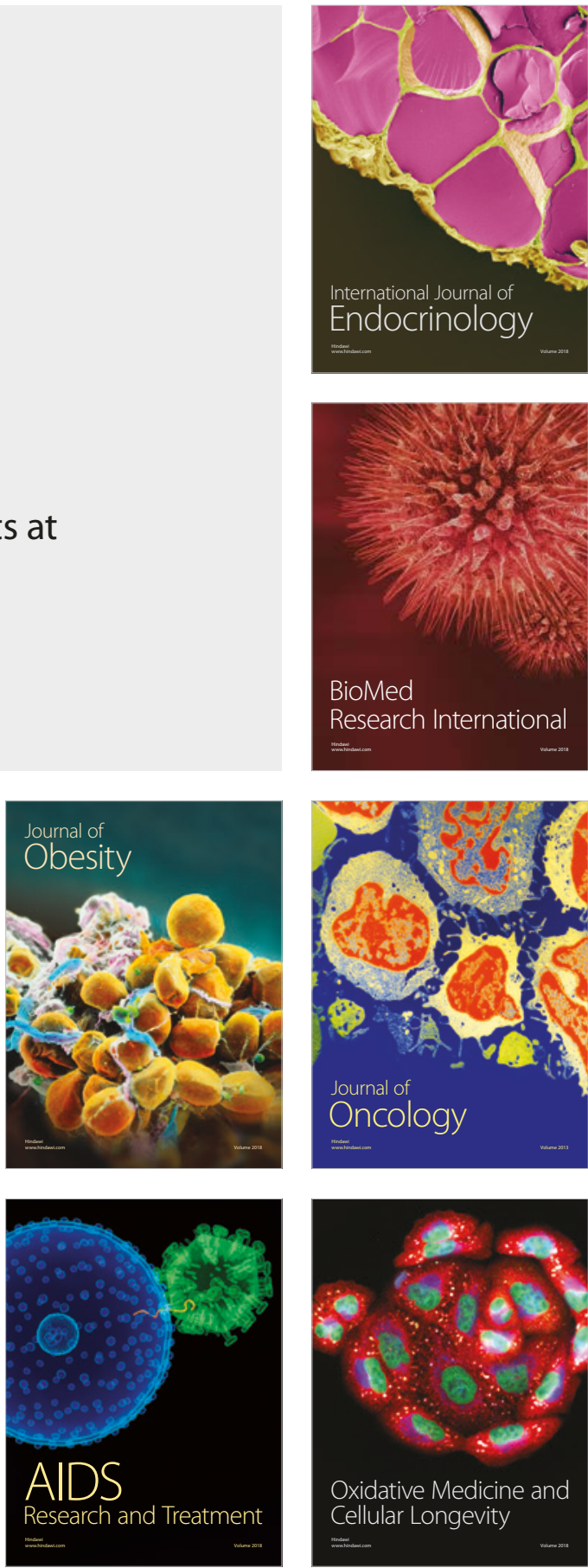